\title{
Characterizing the Impact of COVID-19 on Pre-Exposure Prophylaxis (PrEP) Care
}

\author{
Brooke G. Rogers ${ }^{1,2,3}$ (D) Jun Tao ${ }^{1,2} \cdot$ Michaela Maynard $^{1} \cdot$ Christina Chu $^{1} \cdot$ Elizabeth Silva $^{1} \cdot$ Emily Toma $^{1}$. \\ Katherine $\mathrm{Nagel}^{2} \cdot$ Siena Napoleon ${ }^{1} \cdot$ Philip A. Chan ${ }^{1,2}$
}

Accepted: 4 June 2021 / Published online: 10 June 2021

(c) The Author(s), under exclusive licence to Springer Science+Business Media, LLC, part of Springer Nature 2021

\begin{abstract}
COVID-19 is a public health crisis that has fundamentally altered health care provision. The purpose of this study was to examine the impact of COVID-19 on pre-exposure prophylaxis (PrEP) care. We reviewed all patient records for those who presented for PrEP care at a PrEP program in Providence, Rhode Island from September 1st, 2019 to May 29th, 2020. The number of PrEP encounters decreased but was not significantly different over time ( $p s>.05)$. Patients were still able to access PrEP clinical services during the COVID-19 pandemic. Implementing flexible and timely PrEP delivery approaches in this setting likely minimized the disruption of PrEP care during COVID-19.
\end{abstract}

Keywords Pre-exposure prophylaxis (PrEP) $\cdot$ HIV prevention $\cdot$ Clinical care $\cdot$ COVID-19

\section{Resumen}

COVID-19 es una crisis de salud pública que ha alterado fundamentalmente la prestación de servicios de salud. El propósito de este estudio fue examinar el impacto de COVID-19 en los servicios de la profilaxis preexposición (PrEP). Revisamos todos los registros de pacientes que se presentaron para recibir atención de PrEP en un programa de PrEP en Providence, Rhode Island desde el 1 de septiembre de 2019 hasta el 29 de mayo de 2020. El número de encuentros de PrEP disminuyó pero no fue significativamente diferente con el paso del tiempo (ps > .05). Los pacientes aún pudieron acceder a los servicios clínicos de PrEP durante la pandemia de COVID-19. La implementación de enfoques de entrega de PrEP que eran flexibles y oportunos en este entorno probablemente minimizó la interrupción de la atención médica de la PrEP durante COVID-19.

Philip A. Chan

philip_chan@brown.edu

Brooke G. Rogers

brooke_rogers@brown.edu

Jun Tao

jun_tao@brown.edu

Michaela Maynard

mmaynard2@lifespan.org

Christina Chu

cchu2@lifespan.org

Elizabeth Silva

elizabeth_silva@brown.edu

Emily Toma

etoma@lifespan.org
Katherine Nagel

katherine_nagel@brown.edu

Siena Napoleon

snapoleon@lifespan.org

1 Department of Medicine, Infectious Diseases, The Miriam Hospital, 14 Third Street, Providence, RI 02906, USA

2 Department of Medicine, Warren Alpert Medical School of Brown University, Providence, RI, USA

3 Department of Psychiatry and Human Behavior, Warren Alpert Medical School of Brown University, Providence, RI, USA 


\section{Introduction}

Severe acute respiratory syndrome coronavirus 2 (SARSCoV-2), the cause of coronavirus disease 2019 (COVID-19), has fundamentally shifted several aspects of society including health care [1]. To date, approximately 33.1 million individuals in the United States have received positive confirmatory test for SARS-CoV-2 and approximately 594,000 individuals have died [2]. In the midst of this public health crisis, preventive and primary care as well as routine specialty care has been accessed at lower rates [3-5]. Importantly, this may also include decreased access for sexual health services such as HIV testing and treatment, STI testing and treatment, sexual health examinations, and family planning. However, the extent to which these services have been impacted by COVID-19 is largely unknown.

Pre-exposure prophylaxis (PrEP) is the use of antiretroviral medications to prevent HIV infection. Specifically, tenofovir disoproxil fumarate and emtricitabine (TDF/FTC), and tenofovir alafenamide and emtricitabine (TAF/FTC) are both currently approved by the US Food and Drug Administration for use as a daily oral medication to prevent HIV [6]. Similar to other health behaviors and preventive services, PrEP care during COVID-19 may have been of lower priority to providers and patients due to stay-at-home mandates and/ or experienced disrupted care due to overburdened health care systems. Concerns have been raised about changes to clinical practices in HIV service provision, STI and sexual health services, and the HIV prevention and care continuum [7-9]. Additionally, at least one study reported the immediate impact of COVID-19 on HIV care, which resulted in significantly fewer visits than scheduled, despite efforts to triage cases and offer both in-person and telemedicine [10]. The goal of the current study was to determine the impact of COVID-19 on PrEP care within a clinic based within an academic medical center in New England.

\section{Methods}

\section{Participants and Procedures}

We reviewed medical records for all cisgender male PrEP patients who self-identified as men who have sex with men (MSM) who were engaged in care at the major PrEP program in Rhode Island from September 1, 2019 to May 29, 2020. We included PrEP patients who had been engaged in care and attended PrEP visits during the time phases analysed for this study. Demographic and background information was reviewed from clinical records. Procedures for the review of de-identified clinic data were approved by the The Miriam Hospital Institutional Review Board.

\section{Defining COVID-19 Pandemic Phases}

The first confirmed COVID-19 case in Rhode Island was reported on February 29th, 2020. We defined the "PreCOVID-19 Phase" as starting approximately six months prior to the first case and ending on February 29th, 2020. From March 1st to April 11st, 2020, we observed exponential growth in the number of COVID-19 confirmed cases in Rhode Island and defined this period as the "Evolving Phase." After April 11th, 2020, the number of newly confirmed cases were stable over the time. As such, we used bivariate analysis to compare the average weekly numbers of each type of visit during three phases: (1) Pre-COVID-19 (September 1st, 2019-February 29th, 2020), (2) Evolving COVID-19 Phase (March 1st, 2020-April 11st, 2020), and (3) Plateau Phase (April 12nd, 2020-May 29th, 2020).

\section{Clinic Procedures}

Prior to the COVID-19 pandemic, the clinic had in-person appointments. During the Evolving Phase, the clinic shifted to telehealth and most visits were conducted this way. In the Plateau Phase, the clinic was still seeing most patients through telehealth. During both the Evolving and Plateau phases the clinic providers arranged for in-person appointments as needed. However, the number of patients being seen in-person was significantly limited. Initial and follow up PrEP visits were conducted throughout COVID-19. In accordance with CDC guidance, providers placed initial prescriptions for PrEP once patients had obtained the proper baseline lab work. If patients were seen initially at the STI clinic, they could obtain baseline PrEP labs and then arrange for a telemedicine PrEP appointment to review labs and initiate PrEP. Alternatively, if patients had a telemedicine visit and required labs, lab orders were placed and medication prescribed upon receipt/discussion of results. In-person visits were reserved for those who needed a physical examination, such as those also presenting with STI symptoms.

The standard protocol for providing PrEP prescriptions involves seeing patients approximately every three months. During this time, the clinic made accommodations for those who were not able to attend an appointment and refilled their prescriptions. These flexible care plans extended to lab work as well, which was not required during this time. Patients were encouraged to obtain lab work at the earliest date possible. For follow-up PrEP visits, providers conducted adherence counseling by assessing whether PrEP was appropriate for patients for whom sexual activity had decreased or changed and encouraged patients to re-examine dosing behaviors once stay-at-home mandates were lifted. For PrEP patients who continued to engage in sexual behavior that may place them at risk for HIV (e.g. condomless sex with 
partners with unknown HIV status) and were asymptomatic for bacterial STIs, providers refilled PrEP without requiring the 3-month follow-up labs. Providers counseled patients that forgoing labs for routine PrEP care and STI testing would be temporary, and routine follow-up would continue to be required post COVID-19. Patients who were scheduled to be seen for an in-person appointment were called by clinical staff and were screened for COVID-19 via a phone-based symptom screening protocol prior to their appointment. For laboratory testing patients were instructed to schedule an appointment to limit the number of patients in the clinic/ lab at one time.

\section{Data Analyses}

We used Poisson regression to evaluate the trend of PrEP visit rates throughout the three phases: Pre-COVID-19 Phase, Evolving Phase, and Plateau Phase. The data in Week 13 (Thanksgiving week) and Week 24 (clinic closed due to relocation) were excluded in this analysis. An alpha of 0.05 was used to indicate statistical significance. All analyses were performed in Stata 15.0 (StataCorp LP, College Station, Texas).

\section{Results}

During the period analyzed, our PrEP clinic had a total of 600 visits (85 initial PrEP visits and 515 follow-up PrEP visits). During the Pre-COVID-19 Phase, we had an average of 17 clinic visits per week. Of these visits, three were initial PrEP visits and 14 were follow-up PrEP visits. In the Evolving Phase, we had an average of 15 visits per week, including one initial PrEP visit and 14 follow-up PrEP visits. In the Plateau Phase, we had an average of 13 PrEP visits per week, including one initial PrEP visit and 12 follow-up PrEP visits.

During the Evolving Phase (compared to the PreCOVID-19 Phase), we observed a 6\% [incidence rate ratio (IRR):0.94, 95\% confidence interval (CI): 0.68-1.30, $\mathrm{P}=0.72]$ reduction in the total number of PrEP visits, a $44 \%$ (IRR: $0.56,95 \%$ CI: $0.21-1.50, \mathrm{P}=0.24$ ) reduction in the number of initial PrEP visits, and a 0\% (IRR: $1.00,95 \%$ CI: 0.71-1.41, $\mathrm{P}=0.99)$ decrease in the number of followup PrEP visits. During the COVID-19 Plateau Phase (compared to the Pre-COVID-19 Phase), we observed a $16 \%$ (IRR: $0.84,95 \%$ CI: $0.58-1.20, \mathrm{P}=0.34$ ) reduction in total PrEP visits, a 49\% (IRR: $0.51,95 \%$ CI: $0.18-1.46, \mathrm{P}=0.21$ ) reduction in initial PrEP visits, and a $12 \%$ (IRR: $0.88,95 \%$ CI: $0.60-1.29, \mathrm{P}=0.51$ ) reduction in follow-up PrEP visits. No significant decreases in PrEP visits were observed at any time point. See Fig. 1 for additional details.

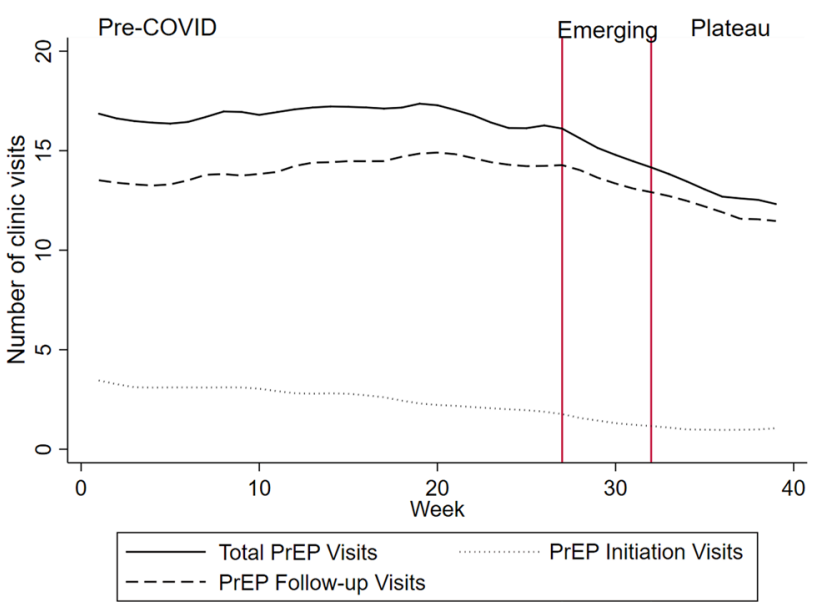

Fig. 1 Number of PrEP clinic visits over time during three distinct phases of COVID-19

\section{Discussion}

This was among the first studies to evaluate changes in access to PrEP care over time at a major PrEP clinic as a result of COVID-19. Although we found a trend of a decrease in both initial and follow-up visits during COVID19 , these differences were not statistically significant. This suggests that patients were still able to access PrEP clinical services during the COVID-19 pandemic. There were several steps the clinic took to ensure continued access to PrEP care during this time. These included allowing individuals to initiate PrEP after obtaining the required lab work and continuing to refill prescriptions for PrEP for those who had been consistently taking it.

While there is limited available published data on the impact of COVID-19 on access and delivery of PrEP, disruptions have been noted in the HIV care continuum for people living with HIV [11]. Additionally, there is emerging data to suggest that similar disruptions have occurred in some PrEP clinics, specifically for patients from marginalized populations [12]. Specifically, research by Krakower and colleagues examined changes in access and utilization of PrEP during COVID-19. PrEP refill lapses increased by $191 \%$, new PrEP starts decreased by $72.1 \%$, and total number of active patients decreased by $18.3 \%$ in their sample. PrEP refill lapses were significantly associated with demographic factors including age, race, ethnicity, and insurance type. Individuals who identified as younger, multiracial/unknown race, Hispanic, and those on public insurance were most likely to experience PrEP refill lapses during this time.

The strengths of our study must be interpreted in light of its limitations. We were limited to a sample of 600 visits within one clinic, which may not be representative of other PrEP programs or clinical settings. Our clinic patients had the ability to access telemedicine and anecdotally reported 
it as favorable when attending appointments with clinic providers given their concerns about COVID-19. Clinics located in larger urban areas or serving lower-income populations could have seen different trends, especially if the transition to telemedicine was more challenging for patients and providers. Additionally, the data presented describe trends in PrEP visits, but does not explore underlying factors associated with these trends. Additional research by our study team is seeking to investigate the impact of COVID-19 on changes in sexual behavior and could provide further insight into our study findings.

\section{Conclusions}

Current research suggests that individuals need continued access to PrEP during COVID-19. PrEP is a critical component of HIV prevention and continuing to provide access to preventive health care during the pandemic is essential. Implementing flexible and timely PrEP delivery approaches may minimize the disruption of PrEP care during the COVID-19 pandemic and could inform responses to address gaps in care caused by future natural disaster or public health emergencies.

Author contributions PAC, JT, and BGR were involved in study design. JT conducted the statistical analyses. BGR wrote the first draft of the manuscript. All authors reviewed and provided feedback on the manuscript.

Funding PAC, JT, SN, and CC are supported by the National Institute of Mental Health (R01MH114657). JT is also supported by the National Institute of Mental Health (K01MH19660). BGR is also supported by Providence/Boston Center for AIDS Research (CFAR), NIH Grant P30AI042853. PAC is on staff at the Rhode Island Department of Health and Rhode Island Public Health Institute.

\section{Declarations}

Conflict of interest BGR receives funding for Gilead Sciences \#INUS-276-5463. There are no other financial or non-financial interests to report.

Ethics approval This study was conducted under a protocol to review retrospective chart data approved by The Miriam Hospital Institutional Review Board.

Consent to participate A waiver of consent was granted for review of de-identified review of clinical data for research purposes.

\section{References}

1. Wosik J, Marat F, Blake C, et al. Telehealth transformation: covid-19 and the rise of virtual care. J Am Med Inform Assoc. 2020;27(6):957-62. https://doi.org/10.1093/jamia/ocaa067.

2. Centers for Disease Control and Prevention. Coronavirus disease 2019 (COVID-19) in the US Atlanta, GA. 2020. Available from: https://www.cdc.gov/coronavirus/2019-ncov/cases-updates/casesin-us.html. Accessed 8 June 2021

3. Prvu Bettger J, Thoumi A, Marquevich V, et al. COVID-19: maintaining essential rehabilitation services across the care continuum. BMJ Glob Health. 2020. https://doi.org/10.1136/ bmjgh-2020-002670.

4. Rubin R. COVID-19's crushing effects on medical practices, some of which might not survive. JAMA. 2020. https://doi.org/10.1001/ jama.2020.11254.

5. Verhoeven V, Tsakitzidis G, Philips H, et al. Impact of the COVID-19 pandemic on the core functions of primary care: will the cure be worse than the disease? A qualitative interview study in Flemish GPs. BMJ. 2020. https://doi.org/10.1136/bmjop en-2020-039674.

6. U.S Food and Drug Administration. FDA approves second drug to prevent HIV infection as part of ongoing efforts to end the HIV epidemic. 2020. Available from: https://www.fda.gov/newsevents/press-announcements/fda-approves-second-drug-preventhiv-infection-part-ongoing-efforts-end-hiv-epidemic. Accessed 10 Sept 2020.

7. Beima-Sofie K, Ortblad KF, Swanson F, et al. Keep It going if you can: HIV service provision for priority populations during the covid-19 pandemic in Seattle, WA. AIDS Behav. 2020. https://doi. org/10.1007/s10461-020-02902-5.

8. Nagendra G, Carnevale $\mathrm{C}, \mathrm{Neu} \mathrm{N}$, et al. The potential impact and availability of sexual health services during the COVID-19 pandemic. Sex Transm. 2020;47(7):434-6. https://doi.org/10.1097/ OLQ.0000000000001198.

9. Napoleon SC, Maynard MA, Almonte A, et al. Considerations for STI clinics during the COVID-19 pandemic. Sex Transm Infect. 2020. https://doi.org/10.1097/OLQ.0000000000001192.

10. Pinto RM, Park S. COVID-19 pandemic disrupts HIV continuum of care and prevention: implications for research and practice concerning community-based organizations and frontline providers. AIDS Behav. 2020. https://doi.org/10.1007/s10461-020-02893-3.

11. Ridgway JP, Schmitt J, Friedman E, et al. HIV care continuum and COVID-19 outcomes among people living with HIV during the COVID-19 pandemic, Chicago, IL. AIDS Behav. 2020. https:// doi.org/10.1007/s10461-020-02905-2.

12. Krakower DS, Solleveld P, Levine K, et al. Impact of COVID-19 on HIV Preexposure Prophylaxis Care at a Boston Community Health Center, NATAP. 2020. Available from: https://www.natap. org/2020/IAC/IAC_75.htm. Accessed 15 Aug 2020.

Publisher's Note Springer Nature remains neutral with regard to jurisdictional claims in published maps and institutional affiliations. 